\title{
Central compact objects and their magnetic fields
}

\author{
Wynn C. G. Ho \\ School of Mathematics, University of Southampton, Southampton, SO17 1BJ, UK \\ email: wynnho@slac.stanford.edu
}

\begin{abstract}
Central compact objects (CCOs) are neutron stars that are found near the center of supernova remnants, and their association with supernova remnants indicates these neutron stars are young $\left(\lesssim 10^{4} \mathrm{yr}\right)$. Here we review the observational properties of CCOs and discuss implications, especially their inferred magnetic fields. X-ray timing and spectral measurements suggest CCOs have relatively weak surface magnetic fields $\left(\sim 10^{10}-10^{11} \mathrm{G}\right)$. We argue that, rather than being created with intrinsically weak fields, CCOs are born with strong fields and we are only seeing a weak surface field that is transitory and evolving. This could imply that CCOs are one manifestation in a unified picture of neutron stars.
\end{abstract}

Keywords. pulsars: general, stars: magnetic field, stars: neutron, supernova remnants

\section{Introduction}

There are a variety of manifestations/classifications of neutron stars. Here we discuss the class dubbed central compact objects (CCOs). CCOs are very loosely defined but are generally characterized by the following observed properties: (1) CCOs are associated with supernova remnants (SNRs) and are therefore young (with ages $<$ a few $\times 10^{4} \mathrm{yr}$ ), (2) CCOs possess thermal X-ray flux that is relatively constant (with X-ray luminosity $L_{\mathrm{X}} \sim 10^{33} \mathrm{ergs} \mathrm{s}^{-1}$ and a spectrum that can be fit by blackbodies from small hot emitting areas), and (3) CCOs have no optical or radio counterpart or pulsar wind nebula (see De Luca 2008; Gotthelf \& Halpern 2008, for observational review, including other CCOs not discussed here; see also Halpern \& Gotthelf 2010).

Only three CCOs currently have a spin period $P$ measured (as well as a measurement or upper limit on the time derivative of spin period $\dot{P}$ ): PSR J0821-4300 in SNR Puppis A has $P=0.112$ s and $\dot{P}<3.5 \times 10^{-16} \mathrm{~s} \mathrm{~s}^{-1}$ (Gotthelf et al. 2010), 1E 1207.4-5209 in SNR PKS 1209-51/52 (also known as G296.5+10.0) has two comparable timing solutions with $P=0.424$ s and $\dot{P}=2.13 \times 10^{-17} \mathrm{~s} \mathrm{~s}^{-1}$ or $1.26 \times 10^{-16} \mathrm{~s} \mathrm{~s}^{-1}$ (Halpern \& Gotthelf 2011), and PSR J1852+0040 in SNR Kesteven 79 has $P=0.105 \mathrm{~s}$ and $\dot{P}=8.68 \times 10^{-18} \mathrm{~s} \mathrm{~s}^{-1}$ (Halpern \& Gotthelf 2010). We hereafter refer to these three CCOs as Puppis A, 1E 1207, and Kes 79, respectively, and only discuss them since we are primarily interested in their magnetic fields.

The spin period derivative values for CCOs are low compared to most radio pulsars (see Fig. 1). Assuming their current $\dot{P}$ is a historical maximum or constant (which may not necessarily be true; see, e.g., Muslimov \& Page 1996; Geppert et al. 1999; Ho 2011; Ho \& Andersson 2012; Pons et al. 2012), then (1) their current spin period is approximately their spin period at birth, $(2)$ their characteristic age $\tau_{\mathrm{c}}(=P / 2 \dot{P}) \gg$ true age, where the true age of Puppis A is $4450 \pm 750 \mathrm{yr}$ (Becker et al. 2012), 1E 1207 is 7 kyr with a factor of 3 uncertainty (Roger et al. 1988), and Kes 79 is 5.4-7.5 kyr (Sun et al. 2004), (3) their X-ray luminosity cannot be powered by rotational energy loss since $L_{\mathrm{X}}>\dot{E}=4 \pi^{2} I \dot{P} / P^{3}$ 


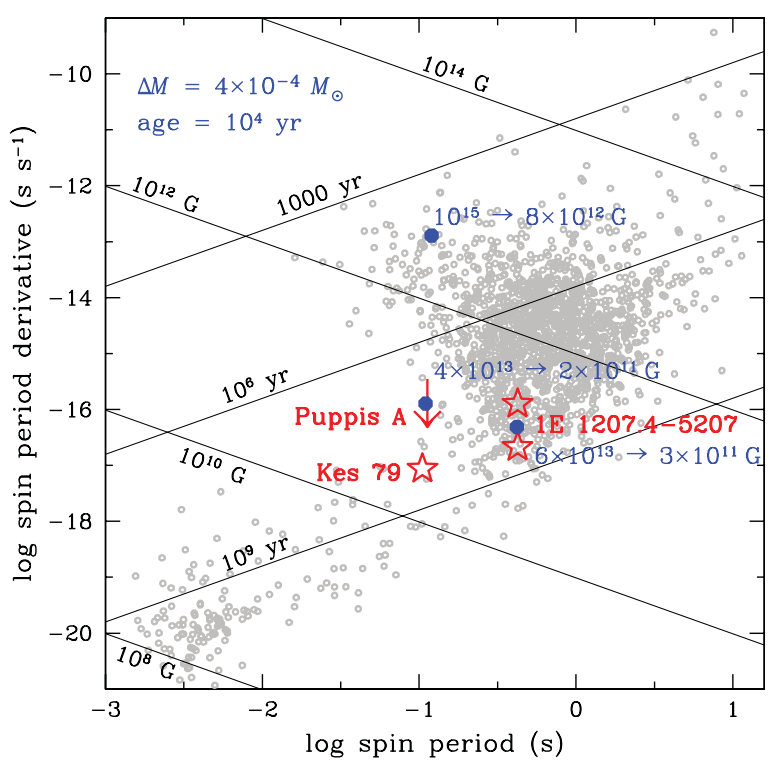

Figure 1. Pulsar spin period $P$ versus spin period time derivative $\dot{P}$. Small open circles are observed values taken from the ATNF Pulsar Catalogue (Manchester et al. 2005). Diagonal lines indicate constant characteristic age $(=P / 2 \dot{P})$ and inferred magnetic field $\left[=3.2 \times 10^{19} \mathrm{G}(P \dot{P})^{1 / 2}\right]$. Stars (or arrow for upper limit on $\dot{P}$ ) denote CCOs at their observed values (1E 1207 has two comparable solutions for $\dot{P}$ ). Large closed circles indicate where these CCOs would be at age $=10^{4} \mathrm{yr}$ if their initial, crust-confined magnetic field (given by the left number) is submerged by an accreted mass $\Delta M / M_{\odot}=4 \times 10^{-4}$; right number is the surface magnetic field at $10^{4} \mathrm{yr}$.

(which could explain their non-detection in radio; see, e.g., Ho 2012), and (4) they possess weak magnetic fields $B \sim 10^{10}-10^{11} \mathrm{G}$ (see next).

\section{Magnetic field of CCOs}

There are two primary methods for determining neutron star magnetic fields. The first involves timing observations, i.e., measuring neutron star spin period $P$ and period derivative $\dot{P}$. Assuming that the rotational energy of the pulsar decreases as a result of the emission of magnetic dipole radiation, the field at the magnetic equator $B_{\mathrm{e}}$ can be inferred from $P$ and $\dot{P}$, i.e., $B_{\mathrm{e}}=3.2 \times 10^{19} \mathrm{G}(P \dot{P})^{1 / 2}$; note that numerical calculations of pulsar magnetospheres yields $B_{\mathrm{e}} \approx 2.6 \times 10^{19} \mathrm{G}\left[P \dot{P} /\left(1+\sin ^{2} \alpha\right)\right]^{1 / 2}$, where $\alpha$ is the angle between the rotation and magnetic axes (Spitkovsky 2006). Using their measured values of $P$ and $\dot{P}$, CCOs have an inferred magnetic field $B \sim 10^{10}-10^{11} \mathrm{G}$ (see Fig. 1).

The second method involves spectral measurements, i.e., identifying features in the neutron star spectrum with particular magnetic processes. Puppis A has a possible emission line at 0.7-0.8 keV (Gotthelf \& Halpern 2009; De Luca et al. 2012), and 1E 1207 has broad absorption lines at 0.7 and $1.4 \mathrm{keV}$ (Sanwal et al. 2002; Bignami et al. 2003). If we assume that a spectral line at energy $E$ is due to electron cyclotron resonance, then the magnetic field is $B=10^{11} \mathrm{G}(E / 1.16 \mathrm{keV})\left(1+z_{\mathrm{g}}\right)$, where $1+z_{\mathrm{g}}=\left(1-2 G M / c^{2} R\right)^{-1 / 2}$ is the gravitational redshift for a neutron star of mass $M$ and radius $R$. The observed lines suggest that CCOs have $B \sim(7-9) \times 10^{10} \mathrm{G}$, in agreement with the fields inferred from timing measurements (spectral fits of another CCO, the 330-yr-old neutron star in SNR Cassiopeia A, suggest it has $B<10^{11} \mathrm{G}$; Ho \& Heinke 2009). 
The magnetic field of CCOs are in contrast to the majority of neutron stars, which possess $B \approx 10^{12}-10^{13} \mathrm{G}$, as can been seen from Fig. 1. Furthermore, from population synthesis studies, neutron star magnetic fields at birth follow a lognormal distribution with an average and width $\sigma$ of $\log B=12.95 \pm 0.55$ in the case of no field decay (Faucher-Giguère \& Kaspi 2006) and $\log B=13.25 \pm 0.6$ when accounting for (modeldependent) field decay (Popov et al. 2010). The natural question is then one of creation versus evolution: Are CCOs born with weak fields or are CCOs born with strong fields but evolve in such a way that they appear to have weak fields at an age of $\lesssim 10^{4} \mathrm{yr}$ ?

Halpern et al. (2007) argue for the former and propose that CCOs are neutron stars that are born spinning slowly. Because of their slow rotation, the dynamo mechanism for magnetic field generation is ineffective, and as a result, CCOs possess weak fields $\left(<10^{11} \mathrm{G}\right)$. However, there appears to be several problems with this creation scenario. First, CCOs do not spin particularly slowly, as illustrated in Fig. 1; this is supported by population synthesis work, which yields a normal distribution for neutron star spin periods at birth with an average and width of $P=0.30 \pm 0.15 \mathrm{~s}$ in the case of no field decay (Faucher-Giguère \& Kaspi 2006) and $P=0.25 \pm 0.1 \mathrm{~s}$ when accounting for field decay (Popov et al. 2010). Second, a birth field $<10^{11} \mathrm{G}$ would require CCOs to be $\gtrsim 4 \sigma$ from the peak of the neutron star distribution, and therefore there should be very few of them relative to the normal pulsar population. But this is counter to their observed numbers. For example, De Luca (2008) finds six CCOs, compared to fourteen radio pulsars, in all known SNRs within $5 \mathrm{kpc}$ (see also Halpern \& Gotthelf 2010), and Kaspi (2010) estimates a CCO birthrate of $\sim 0.0004 \mathrm{yr}^{-1}$ (since all known CCOs are $\lesssim 7$ kyr old) and $\gtrsim 10^{6}$ CCOs in the Galaxy (comparable to the number of strong magnetic field neutron stars). We consider now an alternative to the creation scenario, namely evolution.

\section{Modeling magnetic field evolution}

In the evolution scenario, CCOs are born with strong fields $\left(B>10^{12} \mathrm{G}\right)$, and these fields either decayed rapidly to their current strengths or were buried by an early episode of accretion and are emerging or emerged recently. Magnetic field diffusion and decay conventionally occurs on the Ohmic timescale $\tau_{\mathrm{Ohm}}=4 \pi \sigma_{\mathrm{c}} L^{2} / c^{2} \sim 4 \times$ $10^{5} \mathrm{yr}\left(\sigma_{\mathrm{c}} / 10^{24} \mathrm{~s}^{-1}\right)(L / 1 \mathrm{~km})^{2}$, where $\sigma_{\mathrm{c}}$ is the electrical conductivity, $L$ is the lengthscale over which decay occurs, and $1 \mathrm{~km}$ is the approximate size of the stellar crust (see Goldreich \& Reisenegger 1992). Thus fast decay from $\sim 10^{13} \mathrm{G}$ to $\sim 10^{11} \mathrm{G}$ could only have occurred in CCOs if the field is confined to very shallow layers in the star (Ho 2011).

In Ho (2011), we compare the observed properties of Puppis A, 1E 1207, and Kes 79 to our calculations of the evolution of a buried magnetic field. We assume the field is buried deep beneath the surface (Romani 1990), perhaps by a post-supernova episode of hypercritical accretion (Chevalier 1989; Geppert et al. 1999; Bernal et al. 2010). These fields then diffuse to the surface on the timescale of $10^{2}-10^{4} \mathrm{yr}$, so that only now do we see a surface field $\sim 10^{10}-10^{11} \mathrm{G}$. We solve the induction equation, $\partial B / \partial t=$ $-\nabla \times\left[\left(c^{2} / 4 \pi \sigma_{\mathrm{c}}\right) \nabla \times B\right] \sim B / \tau_{\mathrm{Ohm}}$, in one spatial dimension (see also Muslimov \& Page 1995; Geppert et al. 1999, for non-CCOs), while Viganò \& Pons (2012) perform two-dimensional simulations (and thus are able to account for Hall drift) of burial and emergence of magnetic fields in CCOs. Fig. 1 shows examples of how evolution of an initially submerged magnetic field could change $P$ and $\dot{P}$ for the CCOs. For a field that is confined to the crust, we also find a unique relationship between accreted mass $\Delta M$ and birth magnetic field, with a minimum $\log B \approx 11.4-11.7$. We find that measuring $d B / d t$ or the pulsar braking index would allow a determination of $\Delta M, B$, and the field configuration, e.g., the field is purely in the crust if $d B / d t<0$, while $\Delta M$ is large and the 

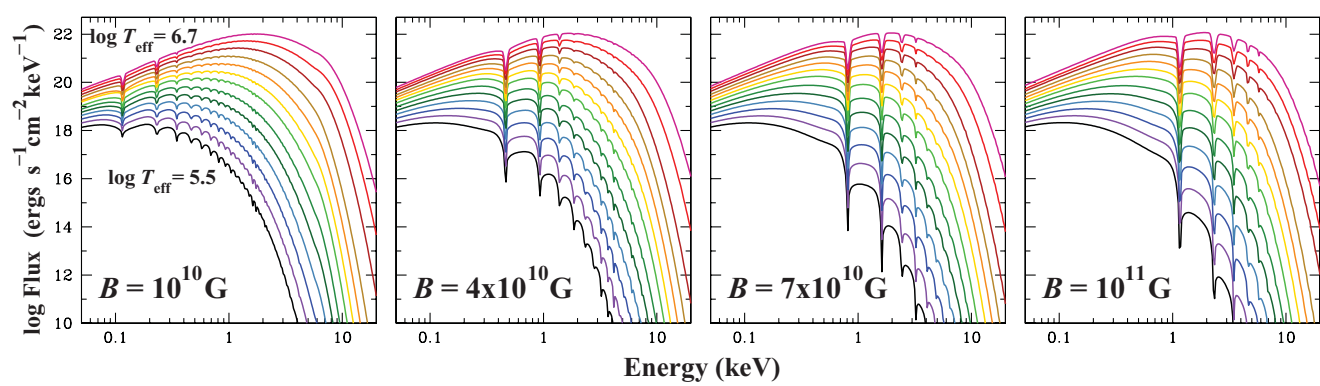

Figure 2. Fully ionized hydrogen atmosphere model spectra for effective temperatures $\log T_{\text {eff }}=5.5-6.7$ and magnetic fields $B=10^{10}, 4 \times 10^{10}, 7 \times 10^{10}, 10^{11} \mathrm{G}$, where the field is oriented parallel to the surface normal.

field is buried deep if $d B / d t>0$ and large. We note that the (candidate) emission line seen in Puppis A has decreased in energy by $10 \%$ in $8.5 \mathrm{yr}$ (De Luca et al. 2012); if this is associated with a decaying magnetic field, then a purely crustal field is implied, although the decay may be too rapid. We also note that optical/IR observations of $1 \mathrm{E} 1207$ place a limit of $\Delta M<10^{-6} M_{\odot}$ on the initial mass of a debris disk (De Luca et al. 2011).

\section{Modeling the magnetized atmosphere spectrum of CCOs}

In addition to advancements in understanding CCO timing properties, progress has been made in modeling their spectra. The observed thermal radiation originates in a thin atmospheric layer (with scale height $\sim 1 \mathrm{~cm}$ ) that covers the stellar surface. The properties of the atmosphere, such as the magnetic field, chemical composition, and radiative opacities, directly determine the characteristics of the observed spectrum (see, e.g., Zavlin 2009, for review). Very importantly, magnetic fields $B>e^{3} m_{\mathrm{e}}^{2} c / \hbar^{3}=2.35 \times$ $10^{9} \mathrm{G}$ significantly increase the binding energy of atoms, molecules, and other bound states, and their abundances can be appreciable in the atmospheres of neutron stars (see Lai 2001, for review). Furthermore, when $B \sim 10^{11} \mathrm{G}\left(T / \mathrm{a}\right.$ few $\left.\times 10^{6} \mathrm{~K}\right)$, models of atmosphere spectra must properly account for quantum and thermal effects in the Gaunt factor or Coulomb logarithm, which give rise to strong cyclotron harmonics in the opacity (Pavlov \& Panov 1976; Pavlov et al. 1980; Potekhin 2010; Suleimanov et al. 2010, 2012). These effects are needed in order to interpret the strong absorption lines seen in $1 \mathrm{E} 1207$ as the result of electron cyclotron resonance in an atmosphere with $B \sim 7 \times 10^{10} \mathrm{G}$, where the observed 0.7 and $1.4 \mathrm{keV}$ lines are the fundamental and first harmonic, respectively (see, e.g., Ho \& Mori 2008, for alternative interpretations).

We construct fully ionized hydrogen atmosphere models using the method described in Ho \& Lai (2001) and using Potekhin \& Chabrier (2003) to calculate Gaunt factors and Suleimanov et al. (2012) to account for thermal effects. Examples of the resulting spectra are shown in Fig. 2. We note that these weak field $\left(B=10^{10}-10^{11} \mathrm{G}\right)$ neutron star atmosphere spectra will be implemented in XSPEC under NSMAX (Ho et al. 2008), while partially ionized hydrogen models will be the subject of future work. The spectra shown in Fig. 2 only describe emission from either a local patch of the stellar surface with a particular effective temperature and magnetic field or a star with a uniform temperature and radial magnetic field of uniform strength. By taking into account surface magnetic field and temperature distributions, we can construct more physical models of emission from neutron stars (see Ho 2007, for details). As an illustration, Fig. 3 shows the phase-resolved model spectra, pulse profile, and pulse fraction. We assume here that 


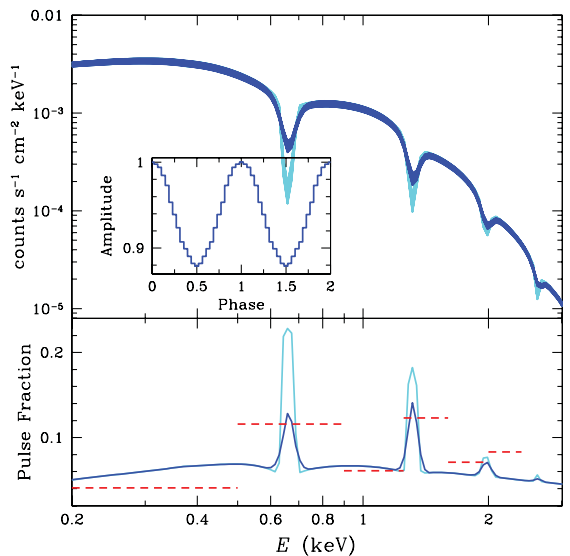

Figure 3. Top: Atmosphere model spectra (raw and convolved with XMM-Newton EPIC-pn energy resolution) at different rotation phases. Inset: Energy-integrated (0.01-10 keV) light curve as a function of rotation phase. Bottom: Pulse fraction as a function of energy, where pulse fraction $=\left(F_{\max }-F_{\min }\right) /\left(F_{\max }+F_{\min }\right)$. The dashed horizontal lines indicate the observed pulse fractions over the given energy range for $1 \mathrm{E} 1207$ (see De Luca et al. 2004).

$1+z_{\mathrm{g}}=1.235$ and angles between rotation and magnetic axes and between rotation axis and observer are $5^{\circ}$ and $25^{\circ}$. The hot spot covers magnetic colatitudes $0-30^{\circ}$ and has effective temperature $T_{\text {eff }}=2 \times 10^{6} \mathrm{G}$ and magnetic field $B=7 \times 10^{10} \mathrm{G}$ that is oriented parallel to the surface normal. The bottom panel shows the pulse fraction in different energy bands that is measured for $1 \mathrm{E} 1207$ (De Luca et al. 2004); what is particularly noteworthy is that the pulse fraction is larger at the spectral lines, and accounting for thermal effects in the model appears to be necessary to achieve these higher pulse fractions (see also Suleimanov et al. 2012).

\section{Discussion}

From timing and spectral studies, CCOs appear to have relatively weak surface magnetic fields $\left(B \approx 10^{10}-10^{11} \mathrm{G}\right)$. The question arises as to whether CCOs are born with inherently weak magnetic fields (creation scenario) or they are born with much stronger fields but these fields were buried and are evolving (evolution scenario). The creation explanation is simple, but as discussed in Sec. 2, there are problems. There are also problems with the evolution scenario (see, e.g., Halpern \& Gotthelf 2010). Nevertheless, evolution of magnetic fields seems natural, and there is evidence in favor of buried magnetic fields in CCOs. For example, Shabaltas \& Lai (2012) construct models with strong toroidal fields $\left(B>10^{14} \mathrm{G}\right)$ in the crust to explain the high pulse fraction of Kes 79 (64 $\pm 2 \%$; Halpern \& Gotthelf 2010). Also Gotthelf et al. (2010) argue that a strong tangential field in the crust can explain the small hot spots seen on Puppis A, and this is confirmed qualitatively with magneto-thermal simulations by Viganò \& Pons (2012).

If CCOs have buried magnetic fields, then this sub-surface field is likely to be $\gtrsim 10^{12} \mathrm{G}$. If burial is shallow, then the surface field is currently decaying. If burial is deep, then the surface field is growing rapidly and could lead to a rapid change in spin parameters. We see from Fig. 1 that CCOs reside in a relatively underpopulated region of $P-\dot{P}$ parameter space (see also Halpern \& Gotthelf 2010; Kaspi 2010). Rapid spin evolution could mean that CCOs are moving out of this region quickly and joining the majority of the pulsar population at longer spin periods, higher $\dot{P}$, and stronger observed magnetic fields. Thus magnetic field evolution could facilitate the unification of CCOs with other classes of neutron stars (see Kaspi 2010; Popov et al. 2010; Ho 2012).

WCGH thanks Alexander Potekhin for discussions, appreciates the use of the computer facilities at KIPAC, and acknowledges support from the RAS and STFC in the UK. 


\section{References}

Becker, W., Prinz, T., Winkler, P. F., \& Petre, R. 2012, ApJ, 755, 141

Bernal, C. G., Lee, W. H., \& Page, D. 2010, Revista Mexicana de Astron. Astrof., 46, 301

Bignami, G. F., Caraveo, P. A., Luca, A. D., \& Mereghetti, S. 2003, Nature, 423, 725

Chevalier, R. A. 1989, ApJ, 346, 847

De Luca, A. 2008, in: C. G. Bassa et al. (eds.), AIP Conf. Proc. Vol. 983, 40 Years of Pulsars (Melville: American Inst. Phys.), p. 311

De Luca, A. et al. 2004, A\&A, 418, 625

De Luca, A. et al. 2011, A\&A, 525, A106

De Luca, A. et al. 2012, MNRAS, 421, L72

Faucher-Giguère, C.-A. \& Kaspi, V. M. 2006, ApJ, 643, 332

Geppert, U., Page, D., \& Zannias, T. 1999, A\&A A, 345, 847

Goldreich, P. \& Reisenegger, A. 1992, ApJ, 395, 250

Gotthelf, E. V. \& Halpern, J. P. 2008, in: C. G. Bassa et al. (eds.), AIP Conf. Proc. Vol. 983, 40 Years of Pulsars (Melville: American Inst. Phys.), p. 320

Gotthelf, E. V. \& Halpern, J. P. 2009, ApJ, 695, L35

Gotthelf, E. V., Perna, R., \& Halpern, J. P. 2010, ApJ, 724, 1316

Halpern, J. P. \& Gotthelf, E. V. 2010, ApJ, 709, 436

Halpern, J. P. \& Gotthelf, E. V. 2011, ApJ, 733, L28

Halpern, J. P., Gotthelf, E. V., Camilo, F., \& Seward, F. D. 2007, ApJ, 665, 1304

Ho, W. C. G. 2007, MNRAS, 380, 71

Ho, W. C. G. 2011, MNRAS, 414, 2567

Ho, W. C. G. 2012, MNRAS, submitted (arXiv:1208.1297)

Ho, W. C. G. \& Andersson, N. 2012, Nature Physics, in press (arXiv:1208.3201)

Ho, W. C. G. \& Heinke, C. O. 2009, Nature, 462, 71

Ho, W. C. G. \& Lai, D. 2001, MNRAS, 327, 1081

Ho, W. C. G. \& Mori, K. 2008, in: C. G. Bassa et al. (eds.), AIP Conf. Proc. Vol. 983, 40 Years of Pulsars (Melville: American Inst. Phys.), p. 340

Ho, W. C. G., Potekhin, A. Y., \& Chabrier, G. 2008, ApJS, 178, 102

Kaspi, V. M. 2010, Proc. National Academy Sci., 16, 7147

Lai, D. 2001, Rev. Mod. Phys., 73, 629

Manchester, R. N., Hobbs, G. B., Teoh, A., \& Hobbs, M. 2005, AJ, 129, 1993

Muslimov, A. \& Page, D. 1995, ApJ, 440, L77

Muslimov, A. \& Page, D. 1996, ApJ, 458, 347

Pavlov, G. G. \& Panov, A. N. 1976, Sov. Phys. JETP, 44, 300

Pavlov, G. G., Shibanov, Yu. A., \& Yakovlev, D. G. 1980, Ap\&SSS, 73, 33

Pons, J. A., Viganò, D., \& Geppert, U. 2012, A\&̈A, 547, A9

Popov, S. B., et al. 2010, MNRAS, 401, 2675

Potekhin, A. Y. 2010, A\& A, 518, A24

Potekhin, A. Y. \& Chabrier, G. 2003, ApJ, 585, 955

Roger, R. S., et al. 1988, ApJ, 332, 940

Romani, R. W. 1990, Nature, 347, 741

Sanwal, D., Pavlov, G. G., Zavlin, V. E., \& Teter, M. A. 2002, ApJ, 574, L61

Shabaltas, N. \& Lai, D. 2012, ApJ, 748, 148

Spitkovsky, A. 2006, ApJ, 648, L51

Suleimanov, V. F., Pavlov, G. G., \& Werner, K. 2010, ApJ, 714, 630

Suleimanov, V. F., Pavlov, G. G., \& Werner, K. 2012, ApJ, 751, 15

Sun, M., Seward, F. D., Smith, R. K., \& Slane, P. O. 2004, ApJ, 605, 742

Viganò, D. \& Pons, J. A. 2012, MNRAS, 425, 2487

Zavlin, V. E. 2009, in: W. Becker (ed.), Ap\&SS Lib. Vol. 357, Neutron Stars and Pulsars (Berlin: Springer-Verlag), p. 181 